\title{
DOES CRANIOPLASTY REDUCE DISABILITY IN CASES WITH POST TRAUMATIC SKULL BONE DEFECTS? OBSERVATIONAL STUDY IN ASSIUT UNIVERSITY HOSPITALS, EGYPT
}

\author{
*Eman S. Shaltout ${ }^{1}$, Wael M. A. Abd El-Ghani ${ }^{2}$, and Wagdi M. Ali ${ }^{3}$, Maha Farid ${ }^{4}$ \\ Department of Forensic Medicine and Clinical Toxicology ${ }^{1}$, Department of Neurosurgery ${ }^{2}$, \\ Department of Plastic Surgery ${ }^{3}$, Faulty of Medicine - Assiut University, Department of \\ Forensic Medicine and Clinical Toxicology ${ }^{4}$, Faulty of Medicine -Helwan University- Egypt. \\ Corresponding author: Eman Salah Shaltout, emansalahshaltout@yahoo.com. \\ Egypt J. Forensic Sci. Appli. Toxicol. Vol 21 (1) \\ Date of submission: 22 March 2020 \\ Revised at: 13 June 2020 \\ Accepted at: 2 July 2020
}

\begin{abstract}
Introduction: Permanent infirmity (disability) is defined as a functional limitation that restricts the ability of a person to perform the daily activities. The incidence of cranial bone defects related to head trauma have increased over the past decades. Cranioplasty could impact the work capability and employability. This study designed to assess the effect of cranioplasty as a method of skull repair on work capability of the patients and their employability. Subjects and Methods: The authors compared the work capability and employment of 35 patients with traumatic cranial bone defects after head trauma treated in the Neurosurgery Department of Assiut University Hospitals, Egypt from January 2013 to January 2018 before and after cranioplasty. Results: Before cranioplasty, 9 patients recorded that they were able to work without limitations according to the WHO classification, 6 patients recorded their ability to work with limitations and 20 recorded being unfit of working. After surgical reconstruction, 26 patients recorded they are able of working without limitations, 5 patients recorded they can work with limitations and 4 recorded being unfit to work for a variety of reasons. Conclusions: Cranioplasty is an efficient protective and cosmetic reconstructive operation, contributing to reduce disability and helping early return to work after traumatic cranial bone defects. This outcome may contribute to improved social and economic state of patients with cranial bone defects.
\end{abstract}

Key words: Cranial defect, Cranioplasty, Employment, Disability, Work capability, Permanent infirmity

\section{INTRODUCTION}

Head injuries are a major cause of death and disability in developed countries and worldwide, mainly caused by road traffic accidents, assaults or falls (Shejoy et al., 2011\& Mohamed Refaat et al., 2019). Cranial defects complicating head injuries has grown during the last hundred years, mostly, these defects occur following the removal of bone segments of compound skull fractures (Bohman \& Schuster, 2013; Wijdicks et al., 2014).

Numerous complications related to cranial defects reported in previous studies (Kurland et al., 2105). The most common complications recorded were subdural hematoma, abscess formation, meningitis, CSF disturbances, unexplained neurological dysfunction including severe headache, dizziness, pain, and discomfort at the craniectomy site (Ashayeri et al., 2016). Such complications may be explained by the fact that once the cranium has been opened, the brain matter, the surrounding meninges and the cranial vessels and nerves are exposed to the external impacts (ex. atmospheric pressure) and become vulnerable with the distortion of its main protective element (Mokri, 2010). 
Previous studies showed that traumatized patients with cranial bone defects suffer from long-term disabilities, were at least independent at home, however, they are likely subjected to the complications. These complications have been linked to increased disability and work incapability (Talan, 2016).

As traditionally used, permanent infirmity or impairment refers to a functional or structural disability of an organ of the body or regarding doing a particular activity, all these terms have linked to reduced work ability of the affected person and therefore rewarding compensations are expected (Eric et al., 2001\& Hafez et al., 2020).

Cranioplasty is an operational repair of a skull bone defect, crucial to cover bone lost following head injury for rebuilding of function, cosmoses, and protection (Abd El-Ghani, 2018). It is a surgical method in which the autologous cranium, made materials (titanium, methyl methacrylate, polyetheretherketone implants, hydroxyapatite, and bioceramics are utilized to repair the head deformity (Shah et al., 2014).

Cranioplasty is more than a corrective repair of cranial bone loss; it is a part of the recovery process taking place after a patient's neurological harm. Reports have shown that cranioplasty may advance the patient's psychological status (Honeybul, 2014).

\section{SUBJECTS AND METHODS}

This study included 35 patients, 29 males and 6 females, extending in age from 14 to 66 years old. Before the patients were enrolled in this study, they were informed about the study and its purpose. They were asked to sign/stamp a written consent to be included as subjects in this study.

Twenty patients were manual (outdoor) workers and fifteen were office (indoor) workers. Their work capability was assessed before the cranioplasty and 6 months after. Before cranioplasty, patients were extensively interviewed in the outpatient clinic to obtain information about their capability to work and the presence or absence of factors limiting their return to their previous occupation. The same questions were asked to the patients 6 months after the cranioplasty to evaluate the effect of the operation on their well-being and working capability.

According to the method described by Abd El-Ghani, (2018), the authors used polymethyl methacrylate which to cover the cranial defect (Figures a \& b).

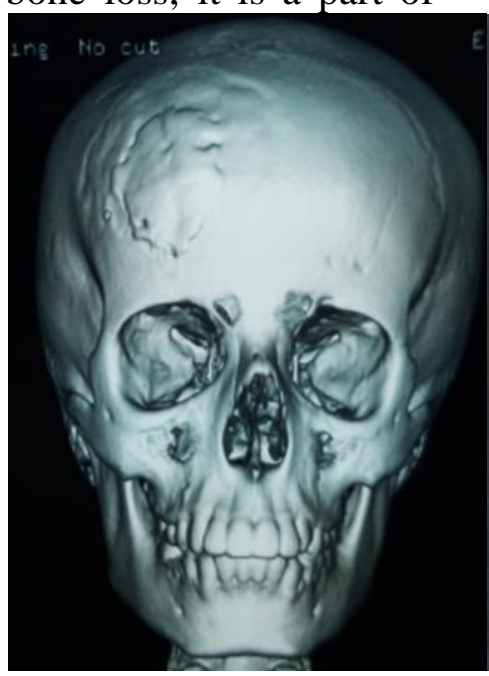

Figure a

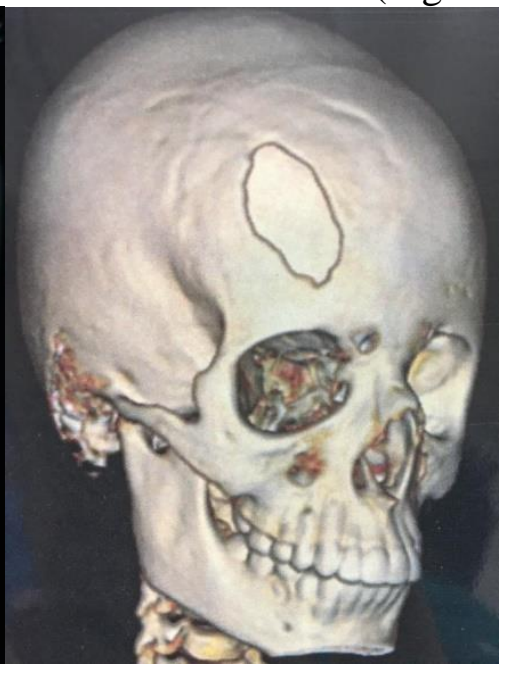

Figure b

Figures (a\&b): 3D CT shows post traumatic frontal skull bone defect of medium size. (a: before $\&$ b: after cranioplasty with poly methyl methacrylate). 
The international classification of impairment, disability and handicaps developed by the WHO, (1980) facilitated the assessment of people according to their capability to work based on his or her wellbeing status and ignoring the age (i.e., whether or not the patient was inside the working age). The classification included 3 groups, first, able of working without limitations including patients with-out physical troubles as paresis, paralysis, aphasia, or other neurological problems. Secondly, patients able of working with limitations; patients with paresis, aphasia, epilepsy, or persistent headaches. Such patients may work in few constrained manners, e.g., in a protected workshop. The last group is patients unfit of working individuals with neurological or other inabilities that prevented their employment. The WHO classification mentioned above, is used to categorize the patients based on their responses to the questionnaire performed in the interview before and after the cranioplasty.

\section{RESULTS}

Table (1): Anatomical site and size of reconstructive cranioplasty in the study subjects.

\begin{tabular}{|c|c|c|}
\hline Location & \multicolumn{2}{|c|}{ Number and size } \\
\hline & Small $(<4 \mathrm{~cm})$ & Medium $(4-8 \mathrm{~cm})$ \\
\hline Frontal & 1 & 8 \\
\hline Parietal & 22 & 2 \\
\hline Temporal & 1 & 35 \\
\hline Total & \multicolumn{2}{|c|}{35} \\
\hline
\end{tabular}

Table (1) describes the sizes of cranioplasty in relation to the anatomical site of the defects. Parietal cranioplasty was done to 22 cases of small size $(<4 \mathrm{~cm})$ and to 8 cases of medium $(4-8 \mathrm{~cm})$ size. Two cases needed frontal cranioplasty, one case was of small size and the other was medium. In the temporal region, one case had small defect and 2 had medium size defects that were corrected by cranioplasty. The time pass between the deformity and the cranioplasty was recorded. The time extended from zero (for prostheses embedded during one operation with craniectomy) to 8 months (with a mean of 4.5 months).

Table (2): Work capability of patients with cranial defects before and 6 months postoperative after cranioplasty as recorded by the patients following outpatient clinic interviews.

\begin{tabular}{|c|c|c|c|c|}
\hline Time & $\begin{array}{c}\text { Capable of } \\
\text { working without } \\
\text { limitations (N/\%) }\end{array}$ & $\begin{array}{c}\text { Capable of } \\
\text { working with } \\
\text { limitations (N/\%) }\end{array}$ & $\begin{array}{c}\text { Incapable of } \\
\text { working. } \\
(\mathrm{N} / \%)\end{array}$ & $\begin{array}{c}\text { Total } \\
(\mathrm{N} / \%)\end{array}$ \\
\hline $\begin{array}{c}\text { Before } \\
\text { cranioplasty }\end{array}$ & $9(25.71 \%)$ & $6(17.14 \%)$ & $20(57.14 \%)$ & $35(100 \%)$ \\
\hline $\begin{array}{c}6 \text { months after } \\
\text { cranioplasty }\end{array}$ & $26(74.28 \%)$ & $5(14.28 \%)$ & $4(11.42 \%)$ & $35(100 \%)$ \\
\hline
\end{tabular}

$\mathrm{N}=$ number.

According to table (2), six months after the cranioplasty, $26 \quad(74.2 \%)$ patients were competent of working without impediments, $5(14.2 \%)$ with impediments, and $4(11.4 \%)$ was unable of working based on the
$\%=$ percentage

patients' recording in the outpatient clinic interviews.

Fourteen (40\%) cases that reported themselves as "unfit" after the craniectomy progressed to "able without limitations" after the 
cranioplasty. In $3(8.5 \%)$ cases, the patients were "able with limitations" after the reconstruction "able without limitations" Two $(5.7 \%)$ cases which

Table 3: Breakdown of the causes of incapability of work before and after cranioplasty as recorded by the patients following outpatient clinic interview.

\begin{tabular}{|c|c|c|c|c|c|c|c|}
\hline \multirow{2}{*}{ Time } & \multicolumn{4}{|c|}{ Presence of neurological deficit } & \multirow{2}{*}{$\begin{array}{c}\text { Fear of } \\
\text { secondary } \\
\text { injury }\end{array}$} & \multirow[t]{2}{*}{$\begin{array}{l}\text { Unsatisfactory } \\
\text { appearance }\end{array}$} & \multirow[t]{2}{*}{ Total } \\
\hline & $\begin{array}{c}\text { Local } \\
\text { pain }\end{array}$ & Epilepsy & Headache & Paresis & & & \\
\hline $\begin{array}{c}\text { Before } \\
\text { cranioplasty }\end{array}$ & 3 & 2 & 2 & 2 & 6 & 5 & 20 \\
\hline $\begin{array}{c}6 \text { months after } \\
\text { cranioplasty }\end{array}$ & 0.0 & 1 & 0.0 & 2 & 0.0 & 1 & 4 \\
\hline
\end{tabular}

As shown in table (3), among the 20 patients who did not work during the period between the craniectomy and the cranioplasty; nine $(45 \%)$ had neurological deficits; two (2) patients were complaining from headache, two (2) patients had post traumatic epilepsy, two (2) were paretic and three (3) had local pain. Additionally, six $(30 \%)$ described the fear of auxiliary damage as the foremost critical reason for not working and five (25\%) were with unsatisfactory appearance.

After the cranioplasty, six out of nine $(66.6 \%)$ patients with the neurological deficits returned to work (for the remining three patients, two were paretic and one was diagnosed with epilepsy, and all three have not recorded any improvement post cranioplasty). Additionally, all the six patients $(100 \%)$ who reported fear of secondary injury returned to work after cranioplasty. Lastly, four out of five ( $80 \%)$ who mention unsatisfactory appearance as the main reason for not returning to work after injury, reported returning to their work contentedly with satisfactory appearance after the cranioplasty.

Patients who did not work were on long-term therapeutic leaves or had been connected for incapacity benefits.

\section{DISCUSSION}

Head injuries are overlooked as contributors to global inequities in health, were 'unfit to work' were "able with limitations" were after the reconstruction. yet the long-term disabilities they frequently produce represent a significant burden (Hoffman et al., 2005).

Each year, around 1.5 million individuals die, and a few millions get crisis treatment as it is the most cause of death and incapacity in individuals beneath 40 years (Wang et al., 2018).

Cranial bone loss is followed by significant neurological dysfunction that happen due to the deficiency of the bone flap, besides, repetitive contamination, electrolyte disturbances, seizures, and cerebrospinal liquid hydrodynamic disturbances, such as hydrocephalus, all of which can have a critical impact on the recovery handle and return to work (Honeybul, 2014). According to several studies, these complications improved after cranioplasty (Ashayeri et al., 2016).

Cranioplasty is crucial to cover bone defects that has been removed after injury or trauma for the purposes of restoring functionality and cosmoses (Abd ElGhani, 2018).

In the current study, major disabilities, and patient's abilities to work return were assessed on 35 patients with cranial defects after trauma and 6 months after cranioplasty by interviewing the patients in the outpatient clinics and recording their answers. Before cranioplasty, the percentage of cases who recorded being able to work without limitations were 
$(25.71 \%),(17.14 \%)$ of cases recorded that they were able to work with limitations and $(57.14 \%)$ of cases recorded not able to work. After the cranioplasty, the majority of patients recorded ability of working without limitations $(74.28 \%)$ of cases, $(14.28 \%)$ of cases recorded ability of working with limitations and only $(11.42 \%)$ of cases recorded themselves as not able to return to work (Table 1).

Kasprzak et al. (2107) divided patients with cranial bone defects according to ability to work into able, able with limitations and unfit to work in compatibility with the WHO's classification (WHO, 1980). Also, according to Kasprzak et al, (2017) improvement in the percentage of work capability were noticed after cranioplasty (Kasprzak et al., 2107), which is inconsistent with the results of the current study.

In this study three reasons were reported by the patients as causes of incapability of work and indicated bone reconstruction: the presence of neurological deficit, the concern of secondary injury and the unsuitable appearance Table (3). Within the analyzed cases, cranioplasty improved the neurological conditions (local pain, epilepsy \& headache) in 6 cases and consequently work ability. Cranial defect reconstruction is essential to avoid a secondary trauma to the brain not secured by the cranium. Another imperative factor is the corrective side. Patient with distorted head appearance by the defect may be unable to return normal social life as reported by some patients that were included in this study.

Honeybul et al., (2013) performed a prospective cohort study that evaluated the neurocognitive parameters in patients 72 hours before and 7 days after cranioplasty, recorded that $4(16 \%)$ out of the 25 patients who were assessed in the study showed improved functional status (Honeybul et al., 2013) .This study had confirmed that recent surgical operation, cranioplasty, to conceal cranial bone defects is significantly advantageous in reducing the percentage of disability, permanent infirmity and costs of financial compensation which is consistent again with the results of the current study.

This concept about the effect of cranioplasty on the patients regaining full work capability needs to be confirmed by other studies with larger sample size and extensive post-operative evaluation.

The primary role of both the neurosurgeon and forensic expert are expected to report on the type of disabilities and severity of impairment caused by traumatic skull bone defects and their effect on the patients' ability to continue to work before and after cranioplasty.

\section{CONCLUSIONS}

Patients who have had skull bone defects facing a challenging problem regarding their ability to return to the work force and cranioplasty is a fundamental surgery that has been proved to improve patients' employment capability. Additionally, cranioplasty has a significant role in bettering patients' neurologic functions and cosmetic appearance. Therefore, cranioplasty is highly recommended whenever it is applicable and/or indicated because of the impact of it correcting the bone defect inflects on the patient's life including his/her socioeconomic status.

\section{REFRENCES}

Abd El-Ghani, WA. (2018): Cranioplasty with polymethyl methacrylate implant: solutions of pitfalls. Egypt $\mathbf{J}$ Neurosurg, 1-4.

Ashayeri, K. Jackson, ME. Huang, J. Brem, H. and Gordon, C. (2016): Syndrome of the Trephined: A Systematic Review. Neurosurgery, 79:525-534.

Bohman, LE. and Schuster, JM. (2013): Decompressive craniectomy for 
management of traumatic brain injury: An update. Curr Neurol Neurosci Rep, 13:392.

Eric, B. Francis, G. Nearkasen, CH. and Lorhandicap, G. (2001): Prevalence of impairments, disabilities, handicaps and quality of life in the general population:a review of recent literature. Bulletin of the World Health Organization, 79:1074-1055.

Hoffman, K. Primack, A. Keusch, G. and Hrynkow, S. (2005): Addressing the growing burden of trauma and injury in low- and middleincome countries. Am J Public Health,95:13-7.

Hafez, A. Elgendy, I. Zamzam, I. Hassan, N. Mohammed Ali, N. Madboly, A. (2020): A Prospective Study of Medico-Legal Aspects of Permanent Infirmity Cases Examined at Forensic Medicine Authority- Cairo Department, Egypt. The Egyptian Journal of Forensic Sciences and Applied Toxicology, 20(1): 65-80.

Honeybul, S. (2014): Neurological susceptibility to a skull defect. Surg Neurol Int, 4:83.

Honeybul, S. Janzen, C. Kruger, K. and Ho, KM. (2013): The impact of cranioplasty on neurological function. Br J Neurosurg, 27:(5):636-641.

Kasprzak, P. Ormezowska, E. and Jaskólski, D. (2017): Cranioplasty as the return-to-work factor - 112 patients with cranial defects treated in the Department of Neurosurgery at the Medical University of Lodz. Int $\mathbf{J}$ Occup Med Environ Health, 30:803809.

Kurland, DB. Khaladj-Ghom, A. Stokum, JA. Carusillo, B. Karimy, JK. and Simard, JM. (2015): Complications Associated with Decompressive Craniectomy: A Systematic Review. Neurocrit Care, 23:292-304.
Mohamed Refaat, R. Haroun, M. Sharf El Din, A. Hussein, A., abd elkader, A. (2019): medico legal aspects of traumatic head injuries in benha university hospital (prospective analytical study). The Egyptian Journal of Forensic Sciences and Applied Toxicology, 19(4):119-145.

Mokri, B. (2010): Orthostatic headaches in the syndrome of the trephined: resolution following cranioplasty. Headache, 50: 1206-1211.

Shah, AM. Jung, H. and Skirboll, S. (2014): Materials used in cranioplasty: a history and analysis. Neurosurg Focus,36: E19.

Shejoy, PJ. Deepak, AM. Satyarthee, GD. Gupta, D. and Sinha, S. (2011): Cranioplasty as a surrogate marker for excellent outcome in severe head injury. Ind J Neu Trau,8:7-10.

Talan, J. (2016): New Evidence to Support Decompressive Craniectomy for Reducing Intracranial Pressure in TBI. Neurology Today, 39-41.

Wang, J. Han, F. Zhao, Q. Xia, B. Jialin Dai, J. Wang, Q. et al.(2018): Clinico-pathological characteristics of traumatic head injury in juvenile, Middle-Aged and Elderly Individuals. International medical journal of experimental and clinical research, 24 : 3256-3264.

WHO, (1980): International classification of impairments, disabilities and handicaps, Geneva, Switzerland.

Wijdicks, EF. Sheth, KN. Carter, BS. Greer, DM. Kasner, SE. and Kimberly, WT. (2014): Recommendations for the management of cerebral and cerebellar infarction with swelling: A statement for healthcare professionals from the American Heart Association/American Stroke Association. Stroke, 45:12221238. 
هل ترقيع الجمجمة يقلل العجز في حالات الفقد العظمي للجمجمة فيما بعد الأصابه؟ دراسة

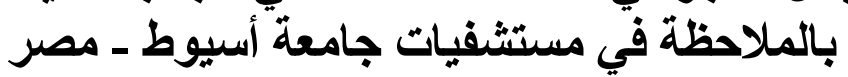

مقدمة: يُعرف العجز الدائم (الإعاقة) بأنه العجز الذبي يقيد قدرة الثخص على العى أداء الأنشطة اليومية.

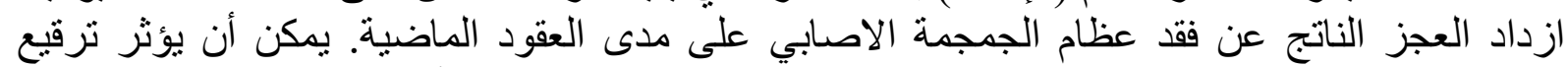

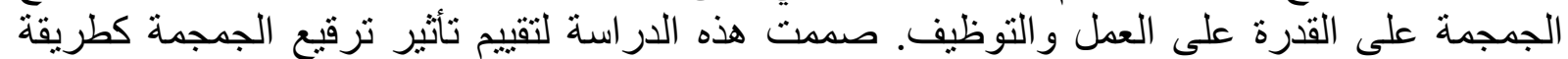

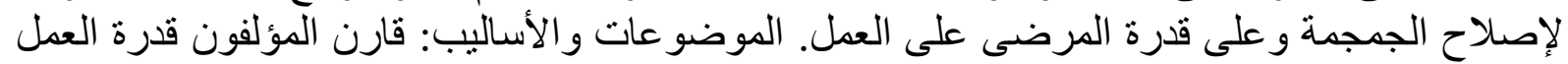

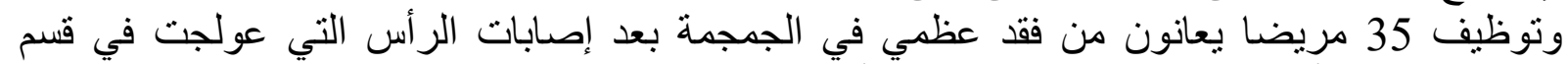

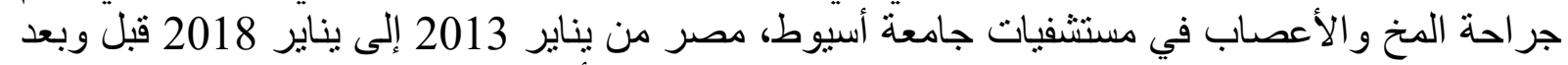

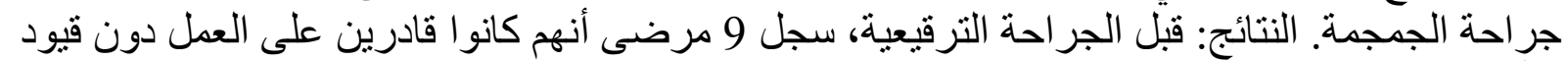

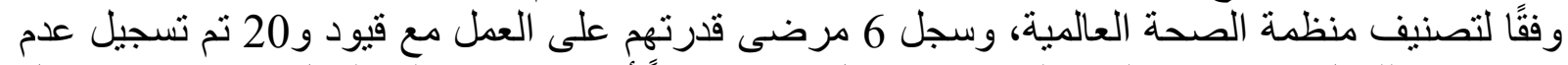

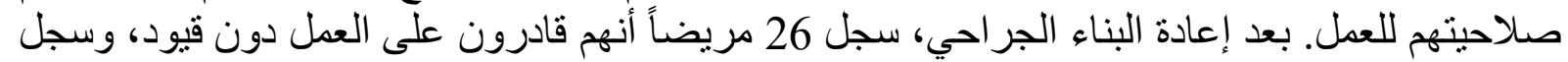

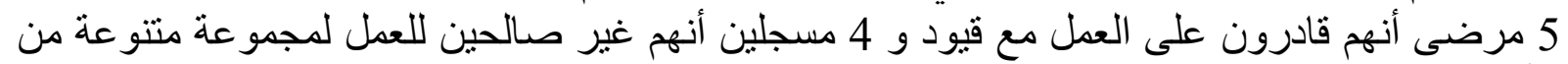

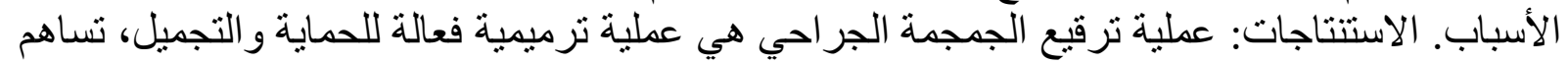

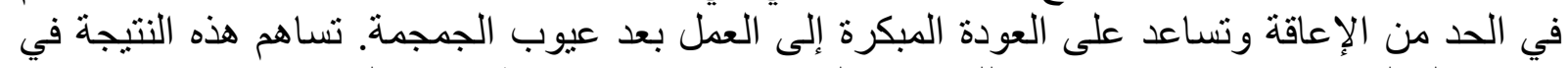

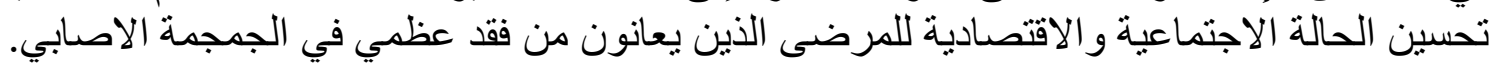

